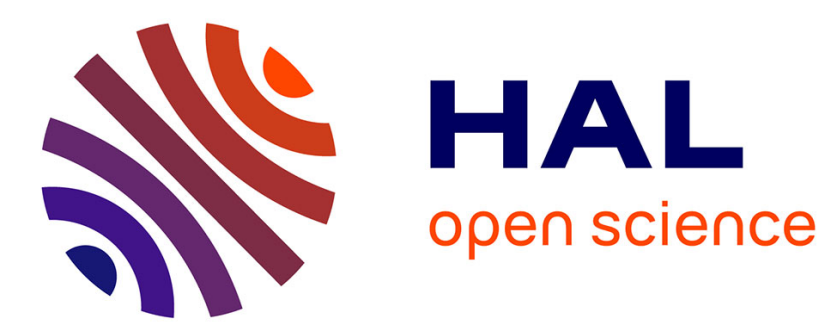

\title{
SHEAR BAND SUSCEPTIBILITY : EFFECT OF HEAT CONDUCTION ON SHEAR BAND FORMATION IN WORK HARDENING MATERIALS
}

T. Wright

\section{- To cite this version:}

T. Wright. SHEAR BAND SUSCEPTIBILITY : EFFECT OF HEAT CONDUCTION ON SHEAR BAND FORMATION IN WORK HARDENING MATERIALS. Journal de Physique IV Proceedings, 1991, 01 (C3), pp.C3-661-C3-665. 10.1051/jp4:1991392 。 jpa-00250538

\section{HAL Id: jpa-00250538 https://hal.science/jpa-00250538}

Submitted on 1 Jan 1991

HAL is a multi-disciplinary open access archive for the deposit and dissemination of scientific research documents, whether they are published or not. The documents may come from teaching and research institutions in France or abroad, or from public or private research centers.
L'archive ouverte pluridisciplinaire HAL, est destinée au dépôt et à la diffusion de documents scientifiques de niveau recherche, publiés ou non, émanant des établissements d'enseignement et de recherche français ou étrangers, des laboratoires publics ou privés. 


\title{
SHEAR BAND SUSCEPTIBILITY : EFFECT OF HEAT CONDUCTION ON SHEAR BAND FORMATION IN WORK HARDENING MATERIALS
}

\author{
T.W. WRIGHT (1) \\ Ballistic Research Laboratory, Abedeen Proving Ground, \\ MD 21005, U.S.A
}

Un modele phénoménologique de matériau rigide plastique avec écrouissage et adoucissement thermique est analysé pour déterminer la susceptibilité de formation des bandes de cisaillement adiabatique. L'accent est mis sur l'influence de la conductivité thermique.

\begin{abstract}
A phenomenological model of a rigid, work hardening, plastic material, with rate hardening and thermal softening, is analysed to determine susceptibility to the formation of adiabatic shear bands. Emphasis is placed on the influence of finite thermal conductivity.
\end{abstract}

\section{1. - Introduction}

Adiabatic shear bands can form in a plastically deforming material when thermal softening due to self induced heating overcomes work hardening and rate hardening so that stress relaxation becomes a consequence of further straining. To investigate this form of plastic instability consider the quasi-static shearing of a finite slab. A simple mathematical model that describes this process may be set down as follows.

$$
\begin{array}{ll}
\text { Momemtum: } & s_{y}=0 \\
\text { Energy: } & \vartheta_{t}=k \vartheta_{Y Y}+s v_{y} \\
\text { Flow Rule: } & s=F(\kappa, \vartheta, \dot{\gamma}) \\
\text { Work Hardening: } & \kappa_{t}=M(k, \vartheta) s v_{y}
\end{array}
$$

with $-1 \leq y \leq+1$ and $0 \leq t<\infty$. In this paper boundary conditions will always be assigned as $v( \pm 1, t)= \pm 1$ and $\vartheta_{y}( \pm 1, t)=0$. The nondimensional dependent variables are shear stress, $s$, temperature above an arbitrary reference level, $\vartheta$, particle velocity, $v$, and work hardening parameter, $\kappa$. Since the material is assumed to be rigid/plastic, the total strain rate, $\dot{\gamma}$, equals the plastic strain rate, and both are equal to the velocity gradient, $v_{y}$. The quasi-static assumption is actually quite a good approximation for nominal strain rates up to several thousand per second if the sheared region is narrow, as in the guage section of a torsional Hopkinson

(1) Current address : Mathematical Institute, University of Oxford, 24-29 st. Giles', GB-Oxford OXI 3LB, Great-Britain. 
bar. Plastic work, $\mathrm{sv}_{\mathrm{Y}^{\prime}}$ acts as a heat source in the energy equation. The flow rule has the properties $F_{\kappa}>0$ for work hardening, $F_{\vartheta}<0$ for thermal softening, and $0<\mathrm{F}_{\dot{\gamma}} \ll \mathrm{F} / \gamma$ for weak rate hardening. The work hardening parameter, $\boldsymbol{\kappa}$, has the status of an internal variable whose rate of evolution is proportional to the rate of plastic work. Its proposed rate equation may seem. unfamiliar, but if $s=\kappa$ in a slow isothermal test, and with $v_{y}=\dot{\gamma}$, the plastic modulus $M(s, 0)$ may be related to the slope of a standard stress/strain test since (1.4) then becomes $\frac{d s}{d \gamma}=M(s, 0) s$. In fact, the evolutionary law for $\kappa$ is versatile enough to mimic most standard representations.

The relationships between the nondimensional (unbarred) and dimensional (barred) quantities are as follows:

$$
\begin{aligned}
& \mathrm{y}=\overline{\mathrm{y}} / \mathrm{h}, \quad \mathrm{t}=\dot{\gamma}_{0} \overline{\mathrm{t}}, \quad \dot{\gamma}_{0}=\mathrm{v}_{0} / \mathrm{h} \\
& \mathrm{v}=\overline{\mathrm{v}} / \mathrm{v}_{0}, \quad \mathrm{~s}=\overline{\mathrm{s}} / \mathrm{S}_{0}, \quad \vartheta=\bar{\rho} \mathrm{c} \bar{\vartheta} / \mathrm{s} \mathrm{s}_{0}, \quad \kappa=\bar{\kappa} / \kappa_{0} \\
& \mathrm{v}_{\mathrm{y}} \equiv \dot{\gamma}=\overline{\mathrm{v}}_{\mathrm{y}} / \dot{\gamma}_{0}, \quad \rho=\bar{\rho} \mathrm{v}_{0}^{2} / \mathrm{s}_{0}, \quad \mathrm{k}=\overline{\mathrm{k}} / \bar{\rho} \mathrm{c} \dot{\gamma}_{0} \mathrm{~h}^{2}
\end{aligned}
$$

where $h, \dot{\gamma}_{0}, V_{0}, S_{0}$, and $\kappa_{0}$ are characteristic values of length, strain rate, particle velocity at the boundary, stress, and work hardening parameter. Density is $\bar{p}$, and heat capacity is $c$.

Equations (1) have homogeneous solutions that depend only on $t$ and are independent of $\mathrm{y}$.

$$
s=s(t), \quad \vartheta=\Theta(t), \quad \kappa=K(t), v_{y}=1
$$

The functions $\Theta(t)$ and $K(t)$ both increase monotonically with time, but $s(t)$ usually will have a single maximum at some positive time.

\section{2. - Linearized Equations}

To determine whether or not the homogeneous solutions are stable against small perturbations, suppose that solutions may be written

$$
\mathrm{s}=\mathrm{S}+\tilde{\mathrm{s}}, \quad \vartheta=\Theta+\tilde{\vartheta}, \quad \kappa=\mathrm{K}+\tilde{\kappa}, \mathrm{v}_{\mathrm{y}}=1+\tilde{\mathrm{v}}_{\mathrm{y}}
$$

and write the linearized equations in the small quantities $\tilde{s}, \tilde{\vartheta}, \tilde{\kappa}$, and $\tilde{v}_{y}$. Since $\tilde{v}( \pm 1, t)=0$ and since it is no restriction to assume that the average initial values of $\kappa$ and $\vartheta$ vanish, $\int_{-1}^{+1} \tilde{\kappa}_{0} d y=$ $\int_{-1}^{+1} \tilde{\vartheta}_{0} d y \equiv 0$, it is easy to show that the stress perturbation and the average values of $\tilde{\kappa}$ and $\tilde{\vartheta}$ all must vanish identically for all time, $\tilde{s}=\int_{-1}^{+1} \tilde{\kappa} d y=\int_{-1}^{+1} \tilde{\vartheta} d y \equiv 0$. Then with a change of dependent variable to $\tilde{\sigma}$ defined by the relationship $M(t) \tilde{\sigma}=\tilde{\kappa}-M(t) \tilde{\vartheta}$ where $M(t)=\exp \left\{\int_{0}^{t} M_{K} S d t\right\}$, the equations reduce to the coupled pair

$$
\begin{aligned}
& \tilde{\vartheta}_{t}=k \tilde{\vartheta}_{Y Y}-\frac{1}{\mathrm{~m}}\left\{\mathrm{~s}_{\vartheta} \tilde{\vartheta}+\mathrm{F}_{\kappa} \mu \tilde{\sigma}\right\} \\
& \tilde{\sigma}_{t}=-\mathrm{k} \frac{\mathrm{M}}{\bar{M}} \tilde{\vartheta}_{Y Y}
\end{aligned}
$$


In (4) note that $\mathrm{S}_{\vartheta}, \mathrm{F}_{\boldsymbol{\kappa}}, \mathbf{M}, \mathcal{M}$, and $\mathrm{m}$ are all functions of $\mathrm{t}$ in general since they are determined from functions which are evaluated on the homogeneous solution. Eurthermore, since $S(t)=F(K(t), \theta(t), 1)$, we have $\mathrm{S}_{\vartheta}=\mathrm{F}_{\kappa} \mathrm{M}+\mathrm{F}_{\vartheta}$, and in the special case $\mathrm{M}_{\vartheta}=0$, it turns out that $M=\mathrm{M} / \mathrm{M}_{0}$.

Since $m$ is a small quantity ( $m \leq .03$ for most metals), it may be supposed that $\tilde{\vartheta}$ and $\tilde{\sigma}$ are $O(1)$ in powers of $\mathrm{m}$ so that the lowest order approximation is

$$
\begin{aligned}
& \tilde{\vartheta}=-\frac{F_{\kappa} M}{S_{\vartheta}} \tilde{\sigma}+O(m) \\
& \tilde{\sigma}_{\tau}=k \tilde{\sigma}_{Y Y}, \quad \text { where } \tau=\int_{0}^{t} \frac{F_{\kappa} M}{S_{\vartheta}} d t
\end{aligned}
$$

The solution is valid as long as $S_{\vartheta}$ remains positive and bounded away from zero. In fact, (5.2) shows that $\tau \rightarrow \infty$ as $t \rightarrow t_{m^{\prime}}$ where $t_{m}$ is the time at which $S_{\vartheta}=0$, and since $\tilde{\sigma} \rightarrow 0$ exponentially at the same time, $\tilde{\vartheta} \rightarrow 0$ there, as well. In other words, the profile of $\tilde{\vartheta}$ tends to become compressed in amplitude as the underlying homogeneous stress approaches its maximum. Furthermore, (5.1) implies that. $\tilde{\vartheta}$ and $\tilde{\sigma}$ are not independent of each other in the main solution even though the initial values of these two variables must be specified independently of each other. This in turn implies the presence of a boundary layer in time after which (5.1) holds. It turns out that the solution varies like $\mathrm{S}^{-1 / \mathrm{m}}$ in the boundary layer, and since $\mathrm{m}$ is small and $\mathrm{S}_{\vartheta}>0$ at $t=0$, the width of the boundary later is approximately $\mathrm{m} / \mathrm{s}_{\vartheta}(0)$. Equation (5.1) may also be written $F_{\kappa}(t) \tilde{\kappa}+F_{\vartheta}(t) \tilde{\vartheta}=O(m)$.

\section{Behavior of Solution Near $s_{\vartheta}=0$.}

As stated above, the solution (5) loses validity near $t=t_{m}$ where $\mathrm{S}_{\vartheta}=0$. Some insight into its behavior there may be found by considering the Fourier components of $\tilde{\vartheta}$ and $\tilde{\sigma}$ in $(4)$, which becomes

$$
\begin{aligned}
& \dot{\tilde{\vartheta}}_{\mathrm{p}}=-\left(\alpha_{\mathrm{p}}^{2}+\frac{\mathrm{S}_{\vartheta}}{\mathrm{m}}\right) \tilde{\vartheta}_{\mathrm{p}}-\frac{F_{\kappa} \mathcal{M}}{\mathrm{m}} \tilde{\sigma}_{\mathrm{p}} \\
& \dot{\tilde{\sigma}}_{\mathrm{p}}=\alpha_{\mathrm{p}}^{2 \mathrm{M} \tilde{\mathcal{\vartheta}}_{\mathrm{p}}}, \text { where } \alpha_{\mathrm{p}}^{2}=\mathrm{k}(\mathrm{p} \pi)^{2} \text { and } \mathrm{p}=1,2,3,4, \ldots
\end{aligned}
$$

For simplicity consider the case where $m=$ const and $M_{\vartheta}=0$ so that $M=M / M_{0}$. Then (6) may be reduced to a single second order ODE 


$$
\begin{aligned}
\ddot{\vartheta}_{p}+A(t) \dot{\tilde{\vartheta}}_{p}+B(t) \tilde{\vartheta}_{p}=0, \text { where } \\
A=S_{\vartheta} / m+\alpha_{p}^{2}-\frac{d}{d t} \ln \left(E_{\kappa} M\right) \\
B=\frac{d}{d t}\left[S_{\vartheta} / m+\alpha_{p}^{2}\right]-\left[S_{\vartheta} / m+\alpha_{p}^{2}\right] \frac{d}{d t} \ln \left(F_{\kappa} M\right)+\frac{F_{\kappa} M}{m} \alpha_{p}^{2}
\end{aligned}
$$

Equation (7) may be put into a standard form by letting $\vartheta_{p}=u w$, where $\mathrm{u}=\exp \left\{-\frac{1}{2} S^{t} A d t^{\prime}\right\}$, and $\mathrm{w}$ satisfies $\ddot{w}-\left(\frac{1}{2} \dot{A}+\frac{1}{4} \mathrm{~A}^{2}-\mathrm{B}\right) \mathrm{w}=0$. That is,

$$
\begin{gathered}
u=e^{-\frac{1}{2} \alpha_{p}^{2} t} S^{-1 / 2 m}\left(\frac{F_{\kappa} M}{F_{\kappa}(0) M(0)}\right)^{1 / 2} \\
\ddot{w}+\left\{-\frac{1}{(2 m)^{2}} \chi^{2}+\frac{1}{2 m}(\dot{\chi}+\phi)\right\} w=0, \text { where } \\
\chi(t)=S_{\vartheta}+m \alpha_{p}^{2}+m_{d t} \frac{d}{d n}\left(F_{\kappa} M\right) \\
\phi(t)=2 \alpha_{p}^{2} F_{\kappa} M
\end{gathered}
$$

Standard WKB treatment of (8.2) with $w=\psi \mathrm{e}^{\omega / 2 m}(1+0(2 \mathrm{~m}))$ leads to

$$
\vartheta_{p} \sim\left\{\begin{array}{l}
\frac{F_{\kappa^{M}}}{\chi} \exp \left\{-\frac{1}{2} \int^{t} \frac{\phi}{\chi} d t^{\prime}\right\} \\
e^{-\alpha_{p}^{2} t} S^{-1 / m} \exp \left\{\frac{1}{2} \int^{t} \frac{\phi}{\chi} d t^{\prime}\right\}
\end{array}\right.
$$

The solution (9) loses validity as $x \rightarrow 0$. Equation (9.1) is actually a refined version of (5), and (9.2) gives rise to the boundary layer. Clearly the finite value of $k$ delays the zero in $x$ past the zero in $S_{\vartheta}$, each Fourier component in turn reaching its corresponding zero in $\chi$.

In the neighborhood of $x=0$ with $\sigma=\sqrt{|\dot{\chi}(0)| / m}\left(t-t_{m}\right),(8.2)$ takes the form

$$
w_{\sigma \sigma}+\left[\frac{1}{2} \frac{\phi(0)}{|\dot{\chi}(0)|}-\frac{1}{2}-\frac{1}{4} \sigma^{2}\right] w=0
$$

whose independent solutions are parabolic cylinder functions

$$
\begin{aligned}
& \mathrm{w}=\mathrm{D}_{\mathrm{n}}(\sigma), \quad \mathrm{n}=\frac{1}{2} \frac{\phi(0)}{|\dot{x}(0)|}-1 \\
& \mathrm{w}=\mathrm{D}_{-\mathrm{n}-1}(i \sigma), \\
& -\mathrm{n}-1=-\frac{1}{2} \frac{\phi(0)}{|\dot{\chi}(0)|}
\end{aligned}
$$

When $\sigma$ is large the asymptotic forms of $D_{n}(\sigma)$ and $D_{-n-1}(\sigma)$ correspond to the forms of (9) when $t-t_{p}=\sqrt{m /|\dot{x}(0)|} \sigma \rightarrow 0$. Thus, the solutions 
in (11) provide the necessary transition through the zeroes of $x$.

Complete details of the above calculations, as well as a proposed scaling law for comparing different materials, will be given in a forthcoming paper. 